\title{
Motor Costs and the Coordination of the Two Arms
}

\author{
Yousef Salimpour and Reza Shadmehr \\ Laboratory for Computational Motor Control, Department of Biomedical Engineering, Johns Hopkins School of Medicine, Baltimore, Maryland 21205
}

We have two arms, many muscles in each arm, and numerous neurons that contribute to their control. How does the brain assign responsibility to each of these potential actors? We considered a bimanual task in which people chose how much force to produce with each arm so that the sum would equal a target. We found that the dominant arm made a greater contribution, but only for specific directions. This was not because the dominant arm was stronger. Rather, it was less noisy. A cost that included unimanual noise and strength accounted for both direction- and handedness-dependent choices that young people made. To test whether there was a causal relationship between unimanual noise and bimanual control, we considered elderly people, whose unimanual noise is comparable in the two arms. We found that, in bimanual control, the elderly showed no preference for their dominant arm. We noninvasively stimulated the motor cortex to produce a change in unimanual strength and noise, and found a corresponding change in bimanual control. Using the noise measurements, we built a neuronal model. The model explained the anisotropic distribution of preferred directions of neurons in the monkey motor cortex and predicted that, in humans, there are changes in the number of these cortical neurons with handedness and aging. Therefore, we found that coordination can be explained by the noise and strength of each effector, where noise may be a reflection of the number of task-related neurons available for control of that effector in the motor cortex.

Key words: aging; handedness; optimal control; signal-dependent noise; tDCS

\section{Introduction}

In tasks in which our two arms cooperate, for example, swing of an axe to split wood, or a backhand to return a tennis serve, the brain is faced with the problem of assigning motor commands to each arm in such a way that the combined actions achieve a goal. This is a form of burden sharing in which there is not a single winner, but continuous cooperation. Indeed, burden sharing is a fundamental problem of any neural computation in motor control: how does the motor system assign responsibility when there are many actors?

For example, suppose one is asked to produce a force along the pulling direction of a muscle. You may imagine that the brain would activate only that muscle in producing the target force. However, this is not the case (Hoffman and Strick, 1999). Other muscles are also activated and contribute to the total force, despite the fact that their pulling directions are not along the target force. As a result, the tuning of a muscle, defined via activation as a function of force direction, is typically broad. Fagg et al. (2002) demonstrated that these tuning functions are consistent with a policy in which the objective is to minimize a cost: given the goal of producing a force, the muscles share the burden in such a way that the sum of squared forces that are produced by each muscle

\footnotetext{
Received July 22, 2013; revised Dec. 2, 2013; accepted Dec. 23, 2013

Author contributions: R.S. designed research; Y.S. performed research; Y.S. and R.S. analyzed data; Y.S. and R.S. wrote the paper.

This work was supported by National Institutes of Health Grant NS078311 and the Human Frontiers Science Program.

The authors declare no competing financial interests.

Correspondence should be addressed to Dr. Yousef Salimpour, 419 Traylor Building, Johns Hopkins School of Medicine, 720 Rutland Avenue, Baltimore, MD 21205. E-mail: ysalimp1@jhmi.edu.

DOI:10.1523/JNEUROSCI.3095-13.2014

Copyright $\odot 2014$ the authors $\quad 0270-6474 / 14 / 341806-13 \$ 15.00 / 0$
}

is minimized. Raising the forces to the power of two, rather than a smaller exponent, produces broad tuning and results in burden sharing rather than winner take all (Shadmehr and Mussa-Ivaldi, 2012).

A similar cost can account for the patterns of motor commands in bimanual tasks. O'Sullivan et al. (2009) considered a task in which one finger from each hand produced a force so that the sum matched a target. They found that, despite the fact that one finger was stronger than the other, the responsibility was shared. The brain assigned a role to each actor based on two properties of that actor: its strength and its variability.

If such a cost underlies how the brain assigns responsibility to the various actors, then any differences in bimanual behavior must arise because of differences in unimanual effort and variance. To test this idea, we first considered the effect of direction of action on bimanual behavior because strength, and presumably effort, varies with direction. We next considered the effect of handedness on bimanual behavior because we expected to see smaller variance for the dominant arm (Kalisch et al., 2006). We then considered the effect of aging on bimanual behavior because aging tends to equalize variance of the two arms (Vaillancourt and Newell, 2003). Finally, we noninvasively stimulated the motor cortex to test whether the resulting changes in unimanual behavior were consistent with changes in bimanual control. Together, we sought to determine whether there was a single principle that could account for bimanual behavior in all these conditions.

\section{Materials and Methods}

A total of 42 volunteers participated in our experiment after giving written, informed consent. These volunteers had no known neurological disease and were naive to the purpose of the experiment. We assessed 
handedness using the Edinburgh Inventory (Oldfield, 1971). The experimental protocol was approved by the Institutional Review Board of the Johns Hopkins School of Medicine.

\section{Behavioral tasks}

Bimanual task. People held the handles of two robotic arms, one in each hand (see Fig. 1A). Each handle housed a force transducer. Pushing on a transducer caused a cursor to move by an amount proportional to the force. The robots counteracted the forces at the handle by a very stiff force field $(\mathrm{K}=2000 \mathrm{~N} / \mathrm{m}, \mathrm{B}=40 \mathrm{~N} . \mathrm{s} / \mathrm{m})$ that maintained each handle in a virtual well, minimizing but not eliminating movements. Position of the cursor, represented by vector $\mathbf{x}$, was proportional to the sum of the force vectors produced by each arm:

$$
\mathbf{x}=a\left(\mathbf{f}_{L}+\mathbf{f}_{R}\right)+\mathbf{x}_{o}
$$

In Equation (1), $\mathbf{x}_{o}$ is the center of the workspace, implying that, when no forces are acting on the transducers, the cursor is at the center. The scaling factor $a$ was set to $0.5 \mathrm{~cm} / \mathrm{N}$, so that, if the two arms together produced $20 \mathrm{~N}$, the cursor was displaced by $10 \mathrm{~cm}$. The cursor was a circle of $0.5 \mathrm{~cm}$ radius. Each trial began with a target placed at the center. The target was a circle of $1 \mathrm{~cm}$ radius. After $1 \mathrm{~s}$, the center target was removed and a goal target appeared in one of 16 locations equally spaced around a circle of radius $10 \mathrm{~cm}$. Therefore, the objective was to produce a $20 \mathrm{~N}$ force vector along the direction of the target. The subject could accomplish this through any combination of forces in the two arms. Our variables of interest were $\mathbf{f}_{L}$ and $\mathbf{f}_{R}$.

The arms were supported in the horizontal plane by an air-sled. We enforced the same posture throughout the experiment by securing the shoulder to the chair and by placing a brace on each wrist. The shoulder and the elbow angles were $\sim 65^{\circ}$ and $60^{\circ}$, respectively. Target directions were presented in a pseudo-random order, and each direction was repeated 10 times, resulting in 160 trials.

Earlier work studied a similar task in which each finger produced a force so that the sum matched a goal (Shim et al., 2005). The novelty of our design was to use direction of the target force, handedness, aging, and brain stimulation to alter the force that the brain assigned to each arm. We asked whether a single principle could account for behavior in the various conditions.

A task to measure unimanual noise. We assumed that the force produced by each arm was a random variable that was affected by noise. We estimated the properties of this noise in a unimanual task. The general setup for the unimanual task was similar to the bimanual task in that a force on the transducer displaced a cursor. On each trial, a target was displayed, corresponding to $10 \mathrm{~N}, 15 \mathrm{~N}$, or $20 \mathrm{~N}$, at a random direction distributed evenly at intervals of $22.5^{\circ}$ around a circle. Visual feedback was provided until the cursor moved inside the target and then eliminated thereafter in that trial. The objective was to maintain the target force for $2 \mathrm{~s}$. We measured the SD of the force during this period. To do so, force was digitally processed using a fifth-order Butterworth filter with a low-pass cutoff of $25 \mathrm{~Hz}$ and then detrended (Craigmile et al., 2005). Subjects performed two trials with each arm for each target force magnitude and direction, for a total of 192 trials.

In this unimanual task, for each target direction $\theta$, the measured force was represented as a scalar quantity that was generated by the following noise model:

$$
\begin{gathered}
f_{R}=u_{R}\left(1+k_{R}(\theta) \phi\right) \\
f_{L}=u_{L}\left(1+k_{L}(\theta) \phi\right) \\
\phi \sim N(0,1)
\end{gathered}
$$

In Equation 2, $u_{R}$ is the motor command to the right arm, and $k_{R}(\theta)$ is the direction-dependent slope of the signal-dependent noise, and $f$ is a scalar, normally distributed random variable with zero mean and variance 1 . To estimate $k_{R}(\theta)$, for each direction $\theta$, we measured the $\mathrm{SD}$ of measured force at each target force and then determined the slope of the SD of measured force versus measured magnitude using linear regression, constraining the intercept to zero. As a result, the unimanual variance mea- surements produced an estimate of signal-dependent noise in each of the 16 directions.

A task to measure unimanual maximum voluntary force. For each target direction, we measured the maximum voluntary force that subjects could produce by each arm and labeled these measurements with scalar functions $m_{R}(\theta)$ and $m_{L}(\theta)$. Participants were instructed to produce the highest possible force in each direction and the maximum value was recorded ( 32 trials, two trials for each direction). The robotic arms were not used in these measurements, as maximum voluntary forces easily exceed capabilities of the robot motors. Rather, a force transducer was mounted at the location of each handle and held in place with bolts that secured the transducer to a rigid frame.

\section{Experimental groups}

Experiment 1: effect of handedness. Two groups of subjects participated in this experiment: a right-handed $(\mathrm{RH})$ group of young volunteers $(n=10$, mean age $\pm \mathrm{SD}$ of $22.2 \pm 2.5$ years, 4 females, Edinburgh laterality index of $88 \pm 13$ ), and a left-handed (LH) group of young volunteers $(n=10,22.6 \pm 3.3$ years, 5 females, Edinburgh laterality index of $-73 \pm 18)$. The experiment began with the Edinburgh handedness survey (Oldfield, 1971). After completion of the survey, subjects performed the unimanual noise task, the bimanual task, and finally the unimanual maximum voluntary force task. To assess the reproducibility of our results, some subjects $(n=2$, one in each group) were repeatedly tested in 10 separate sessions, each session separated by at least one day.

Experiment 2: effect of aging. An RH group of elderly volunteers ( $n=10$, age $59.2 \pm 8$ years, 3 females, Edinburgh laterality index of $76 \pm 22)$ participated in this experiment. The procedures were identical to Experiment 1.

Experiment 3: effect of brain stimulation. We began with a pilot study of two volunteers: one RH (24 years old, male) and the other LH (21 years old, male). Each volunteer participated in a $3 \mathrm{~d}$ study. On each day, they received $2 \mathrm{~mA}$ transcranial direct current ( $\mathrm{tDCS}$ ) stimulation of the motor cortex. The experiment began with placement of the electrodes on the head. The electrodes were two $5 \times 5 \mathrm{~cm}^{2}$ sponges soaked in saline. On each day, we measured bimanual control only, with the aim of quantifying whether stimulation of the motor cortex altered the forces that were produced by each arm. On each day, we considered one of three configurations: the anodal electrode on the dominant motor cortex and the cathodal electrode on the nondominant motor cortex, the anodal electrode on the nondominant motor cortex and the cathodal electrode on the dominant motor cortex, and sham stimulation of the motor cortex. The motor cortex on each hemisphere was localized using the 10-20 EEG system, with one electrode placed on marker C3 (left hemisphere) and the other electrode placed on marker C4 (right hemisphere).

After this pilot study, we recruited a new group of $\mathrm{RH}$ young volunteers who received $2 \mathrm{~mA}$ tDCS stimulation of the motor cortex (age $25.6 \pm 3.7$ years, 5 females, Edinburgh laterality index of $82 \pm 18$ ). The anodal electrode was positioned on the dominant motor cortex, whereas the other electrode was placed on the contralateral motor cortex. After placement of the electrodes, the subjects received sham stimulation in which the current was gradually increased during a $30 \mathrm{~s}$ period, and then returned to zero. After the sham stimulation, the subjects performed the bimanual task. After completion of this task, the current was gradually increased to $2 \mathrm{~mA}$ and maintained there as subjects repeated the bimanual task. This was followed by the unimanual noise task, and the unimanual maximum voluntary force task. Current was delivered through the electrodes using a Phoresor II Auto device (model PM850; IOMED). The stimulation lasted $\sim 25 \mathrm{~min}$.

\section{Mathematical modeling}

We modeled a simplified version of the task in which the motor commands that were produced by each arm were scalars in the same direction as the target:

$$
f^{\star}=u_{L}+u_{R}
$$

In Equation 3, $f^{*}$ is the target force $(20 \mathrm{~N})$ and $u_{L}$ and $u_{R}$ are the forces produced by the right and left arm. Equation 3 represents an ill-posed 
problem for the brain: it is unclear how much force one should produce by each arm in order for the sum to equal the target. People's behavior, however, suggested a consistent and reproducible solution. To account for that behavior, we considered a cost that was composed of two terms: one that penalized noise and one that penalized effort:

$$
J=\frac{1}{2 \operatorname{tr}[K(\theta)]} \mathbf{u}^{T} A K(\theta) \mathbf{u}+\frac{1}{2 \operatorname{tr}[M(\theta)]} \mathbf{u}^{T}(I-A) M(\theta) \mathbf{u}
$$

In the above equation, $t r$ is the trace operator, $\mathbf{u}=\left[\begin{array}{ll}u_{L} & u_{R}\end{array}\right]^{T}, K(\theta)$ is a matrix that describes a quantity related to signal-dependent noise for each arm:

$$
K(\theta)=\left[\begin{array}{cc}
k_{L}^{2}(\theta) & 0 \\
0 & k_{R}^{2}(\theta)
\end{array}\right]
$$

$M(\theta)$ is a matrix that describes a quantity related to maximum voluntary force for each arm:

$$
M(\theta)=\left[\begin{array}{cc}
m_{L}^{-2}(\theta) & 0 \\
0 & m_{R}^{-2}(\theta)
\end{array}\right]
$$

$A$ is a matrix that describes the relative importance of noise and effort in the cost function:

$$
A=\left[\begin{array}{cc}
a_{L} & 0 \\
0 & a_{R}
\end{array}\right]
$$

The mathematical problem is to find the motor commands $\mathbf{u}$ that minimizes the cost in Equation 4, given the constraint in Equation 3. We solved this problem using Lagrange multipliers, which produced a closed form solution for $\mathbf{u}$ as a function of the known quantities $K(\theta), M(\theta)$, and $f^{*}$ :

$$
\begin{aligned}
\mathbf{u}^{\star}=\left(\frac{A K(\theta)}{\operatorname{tr}[K(\theta)]}+\frac{(I-A) M(\theta)}{\operatorname{tr}[M(\theta)]}\right)^{-1} & \mathbf{p}^{T}\left(\mathbf { p } \left(\frac{A K(\theta)}{\operatorname{tr}[K(\theta)]}\right.\right. \\
& \left.\left.+\frac{(I-A) M(\theta)}{\operatorname{tr}[M(\theta)]}\right)^{-1} \mathbf{p}^{T}\right)^{-1} f^{\star}
\end{aligned}
$$

In Equation 8, $\mathbf{p}=\left[\begin{array}{ll}1 & 1\end{array}\right]$. The only unknown quantity in Equation 8 is matrix $A$, which represents the weight of noise for each arm (we assume that the weight of effort is $I-A$ ). We found the quantity $A$ that minimized the sum of squared errors across directions $i=1, \ldots, 16$, between the observed and predicted motor commands $\Sigma_{i}\left(\mathbf{u}_{i}-\mathbf{u}_{i}^{*}\right)^{T}\left(\mathbf{u}_{i}-\mathbf{u}_{i}^{*}\right)$. We used bootstrapping to select subjects in each group, with replacement, and fitted the model to the average group data. We then repeated the procedure to find the distribution associated with the two unknown parameters $a_{L}$ and $a_{R}$.

In summary, we built a model to account for the choices that people made in the bimanual task. These choices consisted of the forces produced by the left and right arms in various directions. Our model had two unknown variables, $a_{L}$ and $a_{R}$ (the two elements of matrix $A$ ).

\section{Relating signal-dependent noise in the arm to distribution of} neurons in the motor cortex

Although the origin of signal-dependent noise in force production is poorly understood, the available evidence suggests that it is of neural origin (Jones et al., 2002), reflecting the number of neurons that are contributing to production of force (Galganski et al., 1993). Suppose that, in the unimanual task, when force $f$ is produced at target direction $\theta$, there are $n$ neurons that are contributing to production of this force, each with discharge rate $d_{i}$ :

$$
f(\theta)=\sum_{i=1}^{n(\theta)} d_{i}
$$

In Equation 9, the number of neurons is direction-dependent, as reflected in the variable $n(\theta)$, which implies that there may be more neu- rons that contribute to force in one direction than another. Let us further suppose that the noise in these neurons is signal-dependent, with a variance that grows quadratically as a function of the mean discharge rate $\bar{d}_{i}$, with a slope specified by $p^{2}$ :

$$
d_{i} \sim N\left(\bar{d}_{i}, p^{2} \bar{d}_{i}^{2}\right)
$$

If we assume that the expected value of the discharge rate is related to the force:

$$
E\left[d_{i}\right]=\frac{f}{n(\theta)}
$$

then the SD of the resulting force is related to the noise in these neurons with the following equation:

$$
S D[f]=\frac{p E[f]}{\sqrt{n(\theta)}}
$$

Equation 12 implies that, as the number of neurons increase, the SD of noise decreases. In each of our experiments, we measured the SD of force for each arm at each direction and modeled it as follows:

$$
S D[f]=k(\theta) E[f]
$$

From Equations 12 and 13, we see that the number of neurons that contribute to force in each direction is inversely proportional to the squared slope of the signal-dependent noise in force:

$$
n(\theta)=\frac{p^{2}}{k^{2}}
$$

Because variable $p$ is an arbitrary constant, in our simulations we set it to 1 and used Equation 14 to estimate the direction-dependent distribution of neurons that are contributing to force production for each arm in each group of subjects.

\section{Statistical analysis}

Our data consisted of force measures taken from each arm along 16 directions. In the bimanual task, we used the fraction of the total force produced by a given arm as the measure along each direction and then computed the effect of direction and group using repeated-measures ANOVA (Mathematica 8, Wolfram). To summarize the data across directions, we computed a laterality index. For example, a laterality index for the force produced by the right and left arms in the bimanual task was defined as follows:

$$
L=\frac{\sum_{i=1}^{16} f_{R}^{(i)}-f_{L}^{(i)}}{\sum_{i=1}^{16} f_{R}^{(i)}+f_{L}^{(i)}}
$$

In Equation 15, a positive laterality index implies that the subject used their right arm more than left.

\section{Signal-dependent noise in joint coordinates}

We had measured signal-dependent noise of unimanual force in Cartesian space. We wanted to represent this in joint space (i.e., in terms of torques not forces). We began with the assumption that force in Cartesian space was a vector in direction $\theta$ with magnitude $r$ and the following noise properties:

$$
\begin{gathered}
\mathbf{f}=r\left[\begin{array}{c}
\cos (\theta) \\
\sin (\theta)
\end{array}\right] \\
r \sim N\left(\bar{r}, k^{2}(\theta) \bar{r}^{2}\right)
\end{gathered}
$$

In Equation 16, $\bar{r}$ is the expected value of $r$, and $k(\theta)$ is the directiondependent signal-dependent noise in Cartesian space. We were inter- 
ested in estimating this noise in joint space. In joint space, the force vector becomes a torque:

$$
\tau=J^{T} \mathbf{f}=r J^{T}\left[\begin{array}{c}
\cos (\theta) \\
\sin (\theta)
\end{array}\right]
$$

In Equation 17, $J$ is the Jacobian matrix $\frac{d \mathrm{x}}{d \theta}$ for the left or the right arm, and the superscript $T$ is the transpose operator. The expected value and variance of this torque vector are as follows:

$$
\begin{gathered}
E[\tau]=\bar{r} J^{T}\left[\begin{array}{c}
\cos (\theta) \\
\sin (\theta)
\end{array}\right] \\
\operatorname{var}[\tau]=k^{2}(\theta) \bar{r}^{2} J^{T}\left[\begin{array}{c}
\cos (\theta) \\
\sin (\theta)
\end{array}\right][\cos (\theta) \sin (\theta)] J
\end{gathered}
$$

The variance equation can be simplified as follows:

$$
\operatorname{var}[\tau]=k^{2}(\theta) E[\tau] E[\tau]^{T}
$$

From Equation 19, we infer that, if the SD of noise grows linearly as a function of force (as in Eq. 16), then the SD of torque also grows linearly as a function of torque. Therefore, to estimate noise in joint space, we first estimated $k(\theta)$ by fitting an interpolating spline function to the measured values in Cartesian space. This made it so that, for any direction $\theta$ of unimanual force in Cartesian space, we could estimate $k(\theta)$. Next, for a given torque vector $t$, we first computed the corresponding force vector $\mathbf{f}$ using the equation: $\mathbf{f}=J^{-T} \tau$, where the superscript $-T$ is the inverse transpose operator (we assumed the arm configuration shown in Fig. 8A, with upper arm length of $28 \mathrm{~cm}$ and forearm length of $32 \mathrm{~cm}$ ). Next, we computed the direction of $\mathbf{f}$ in Cartesian space, resulting in angle $\theta$. For that direction, we computed $k(\theta)$, which according to Equation 19 represents the signal-dependent noise for the torque vector $t$.

\section{Results}

We considered a task in which people chose how much force to produce with each arm, with the constraint that the sum had to match an instructed magnitude and direction. We imagined that this choice may be related to the strength and variability of each arm. To test this idea, in Experiment 1 we asked whether the bimanual choice was consistent across repeated measurements, whether it was affected by direction of target force (because strength, and presumably effort, are direction-dependent), and whether it was affected by handedness (because the dominant arm is generally less variable). In Experiment 2, we considered the effect of aging (which affects both strength and variability). The results of these two experiments suggested that unimanual noise and strength were important factors that affected people's choices in the bimanual task. We considered the possibility that these factors were related to the properties of the neuronal population that was recruited in the motor cortex. To test this idea, in Experiment 3 we used tDCS to bilaterally stimulate this region of the brain, increasing the excitability of one hemisphere while decreasing the excitability of the other (Mordillo-Mateos et al., 2012).

\section{Experiment 1: handedness and bimanual control}

Results of the bimanual task for two representative subjects are shown in Figure $1 B$. For ease of presentation, for each target direction we have projected the force vector produced by each arm along the target direction and plotted the resulting magnitude. We observed that, for the LH subject, contribution of the left arm was usually greater than the right arm, but not for all directions. For example, for the target at $0^{\circ}$, the left arm of the $\mathrm{LH}$ subject produced three times as much force as the right arm, but for the target at $180^{\circ}$ the contributions of the two arms were
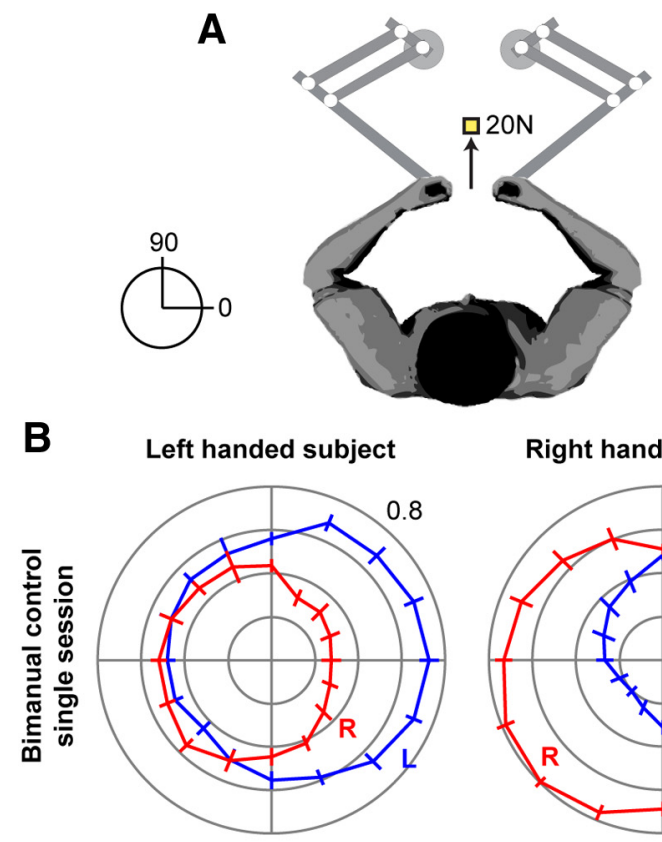

Right handed subject
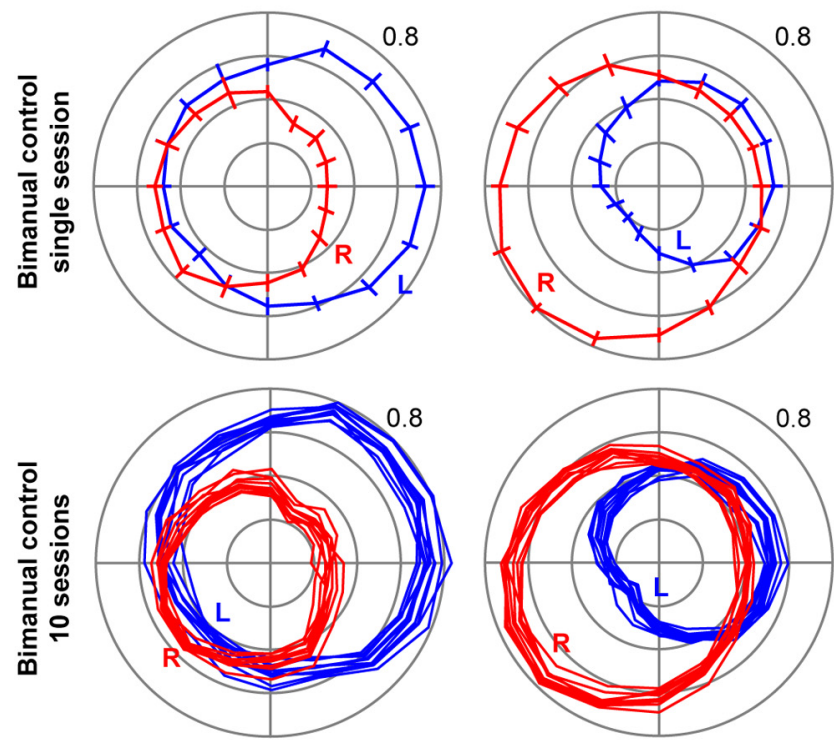

C
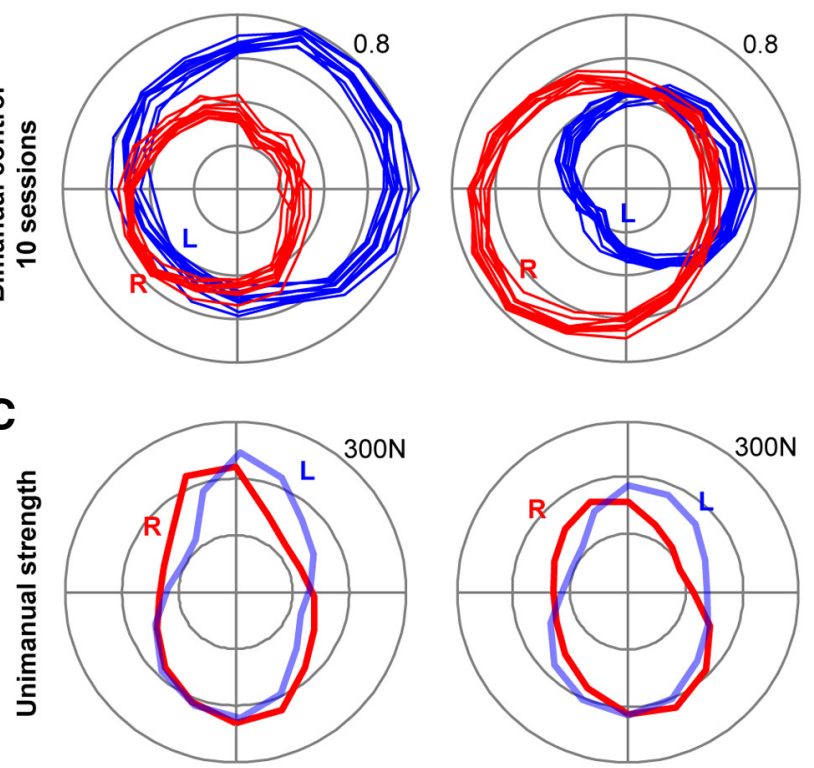

Figure 1. Experimental paradigm and performance of representative subjects. $A$, Volunteers held a force transducer in each hand. The transducers were housed in the handles of two robotic arms. The task was to produce forces with the two arms so that the sum of the two forces would be a vector that displaced a cursor to a target at $20 \mathrm{~N}$. The targets were randomly selected from 16 directions, uniformly distributed around a circle. The task was nearly but not absolutely isometric, as the robotic arms were programmed to produce a very stiff "well" that strongly resisted but did not completely eliminate movement. $\boldsymbol{B}$. The force produced by each arm, represented as the fraction of total force produced by the two arms. For example, in the $\mathrm{LH}$ subject, the target force of $20 \mathrm{~N}$ at $0^{\circ}$ resulted in production of $\sim 14 \mathrm{~N}$ by the left arm and $6 \mathrm{~N}$ by the right arm. In the RH subject, the same target force resulted in production of $11 \mathrm{~N}$ in the left arm and $9 \mathrm{~N}$ in the right arm. Top row, The across-trial, within-session variability of forces. Error bars indicate SD. Bottom row, Data from the same two subjects collected over 10 separate days (one line per day). The choices that the subjects made were highly consistent in repeated measures. C, Unimanual MVF as a function of direction. Whereas the MVF patterns of the two arms are not different between the subjects, the choices that they made in bimanual control appear quite different.

nearly the same. This pattern was substantially different for the $\mathrm{RH}$ subject. In the $\mathrm{RH}$ subject, the contributions of the right arm was greater than the left arm, but not for all directions. For the target at $180^{\circ}$ the right arm produced approximately three times 
as much force as the left arm, but for the target at $0^{\circ}$ the contributions of the two arms were about the same. These choices were consistent both within a session and across sessions, as illustrated by the within-session error bars in the first row of Figure $1 B$, and the across-session measurements in the second row of Figure $1 B$ (these data are from measurements on 10 separate days from each subject). Therefore, when the subjects were asked to produce a target force, their brain made a consistent choice regarding the contribution of each arm. This choice was a function of direction of the target, as well as handedness.

A simple hypothesis regarding the origin of these patterns is that they arise from unimanual strength of each arm. Such a hypothesis would predict that the LH subject has a stronger left arm, and the RH subject has a stronger right arm. To check for this, we measured the maximum voluntary force (MVF) in each arm. The results are plotted in Figure $1 C$. We found that, in the LH subject, the left arm did not appear stronger than the right arm, and in the RH subject, the right arm did not appear stronger than the left arm. Therefore, in these subjects, strength of the arms did not appear to account, at least by itself, for the choices that the brain made in assigning motor commands during bimanual control.

Group data in the bimanual task are plotted in Figure 2. In Figure $2 A$, we have plotted the force vector produced by each arm, representing it as a variance-covariance ellipse in which the center location corresponds to the mean of the force vector. We found that, in general, when asked to produce a goal force, each arm produced a force vector that was nearly parallel with the goal force. To quantify the angular distance between the goal force and the force in each arm, we measured the average absolute value of the angle between these forces. We found that, in the $\mathrm{LH}$ group, the force in the left and right arms was $8.1 \pm 0.8^{\circ}$ and $10.3 \pm 1.5^{\circ}$ apart from the goal force (mean \pm SEM), whereas in the $\mathrm{RH}$ group, the force in the left and right arms was $10.1 \pm 1.6^{\circ}$ and $8.4 \pm 0.9^{\circ}$ apart from the goal force. The dominant and the nondominant arms did not differ in their deviations within each group (LH group, $t$ test, $p=0.16$; $\mathrm{RH}$ group, $p=0.08$ ), although there was a trend toward the dominant arm having a smaller deviation.

Given the small angular distance between the force vector produced by each arm and the goal vector, for each target direction we used as our proxy for force a scalar measure: the projection of the force vector produced by each arm onto the goal vector. The result of this representation is shown in Figure $2 B$, where the error bars are now SEM. We see that, in the LH group, the left arm generally produced a greater force when the target was to the right, whereas in the RH group the right arm generally produced a greater force when the target was to the left. We quantified behavior in each direction by subtracting the force produced by the left arm from the right arm and then performed a repeated-measure ANOVA with direction as the repeated measure and handedness as the group factor. We found a main effect of handedness $\left(F_{(1,18)}=18.4, p=0.0004\right)$, a main effect of direction $\left(F_{(15,270)}=24, p<10^{-5}\right)$, and no interaction $\left(F_{(15,270)}=0.7, p=0.81\right)$. Therefore, direction and handedness both significantly affected the choices that people made in the bimanual task.

To summarize these data, we used two measures. Our first measure was the average magnitude of force produced by each arm across all directions. This measure is shown in Figure 2D (labeled force). A one-way ANOVA on these data is equivalent to the group main effect reported in the repeated-measure ANOVA above. Our second measure was a laterality index (Eq. 9). The laterality index is bounded by 1 and -1 , indicating preference for use of the right arm or left arm, respectively. This measure is shown in Figure 2E. The LH group showed a preference for the left arm, whereas the RH group showed a preference for the right arm. A one-way ANOVA on these data is also equivalent to the group main effect reported in the repeated measure ANOVA above.

\section{Handedness implies less noise, not greater strength}

The MVF data for the subjects in Experiment 1 are summarized in Figure $2 C$. As has been reported before, the maximum force that one can produce with each arm is along a direction from the hand to the shoulder joint (Jan Nijhof and Gabriel, 2006). We quantified relative strength of each arm in each direction by subtracting the MVF produced by the left $\operatorname{arm} m_{L}(\theta)$ from the right $\operatorname{arm} m_{R}(\theta)$, and then performed an ANOVA with direction as the within-subject repeated measure and handedness as the between-subject group factor. We observed a main effect of direction $\left(F_{(15,270)}=13.9, p=0.0002\right)$, but no effects of group $\left(F_{(1,18)}=0.08, p=0.77\right)$ or interaction $\left(F_{(15,270)}=0.32, p=\right.$ $0.99)$. Therefore, the dominant arm was not stronger than the nondominant arm (Fig. 2E). Rather, the MVF in each arm was direction-dependent.

We measured noise in the motor commands that were sent to each arm by testing the subjects in a unimanual task. In this task, the subjects were asked to maintain a target force without visual feedback. We measured the SD of force at each target force and plotted the results in Figure $3 A$. The warmer colors are associated with greater SD of force. To analyze these data, we first asked whether SD of force depended on force magnitude. For each subject and each force magnitude, we averaged across directions and then performed an ANOVA with force magnitude as the within-arm repeated measure and the dominant and nondominant arms as the between-arm factor. We found a significant main effect of force magnitude $\left(F_{(2,36)}=28.5, p=5.3 \times 10^{-6}\right)$ and a force-magnitude by arm interaction $\left(F_{(2,36)}=5.06, p=\right.$ 0.012). These data are plotted in Figure $3 A$ (right). SD of force increased with magnitude of force. However, the rate of increase was larger in the nondominant arm compared with the dominant arm.

Next, for each subject, we estimated the slope of the relationship between SD of force and force magnitude for each direction and each arm, resulting in signal-dependent noise variables $k_{L}(\theta)$ and $k_{R}(\theta)$. These variables are plotted for the $\mathrm{LH}$ and $\mathrm{RH}$ groups in Figure $3 B$ (left column, labeled Cartesian space). The area of each ellipse corresponds to the average signal-dependent noise in that arm. We see that, for both groups, the dominant arm has a smaller ellipse. Furthermore, the ellipses have a specific orientation, implying that there are specific directions for which the noise is smaller. To quantify these patterns, we performed an ANOVA on the variable $k_{R}(\theta)-k_{L}(\theta)$ with direction as the within-subject repeated measure (in Cartesian coordinates), and $\mathrm{LH}$ and $\mathrm{RH}$ groups as the between-subject factor. We found a significant main effect of direction $\left(F_{(15,270)}=8.8, p=0.003\right)$ and a significant main effect of group $\left(F_{(1,18)}=12.3, p=0.0025\right)$. In a post hoc analysis, we collapsed the signal-dependent noise across directions for each arm and each subject, as shown in Figure $3 C$, and then tested whether the average signal-dependent noise in the dominant arm was different from the nondominant arm. A $t$ test revealed a significant effect of handedness $\left(t_{(19)}=-3.43, p=0.0028\right)$. Therefore, signal-dependent noise was direction-dependent in Cartesian space and larger in the nondominant arm. 


\section{A Left handed group}
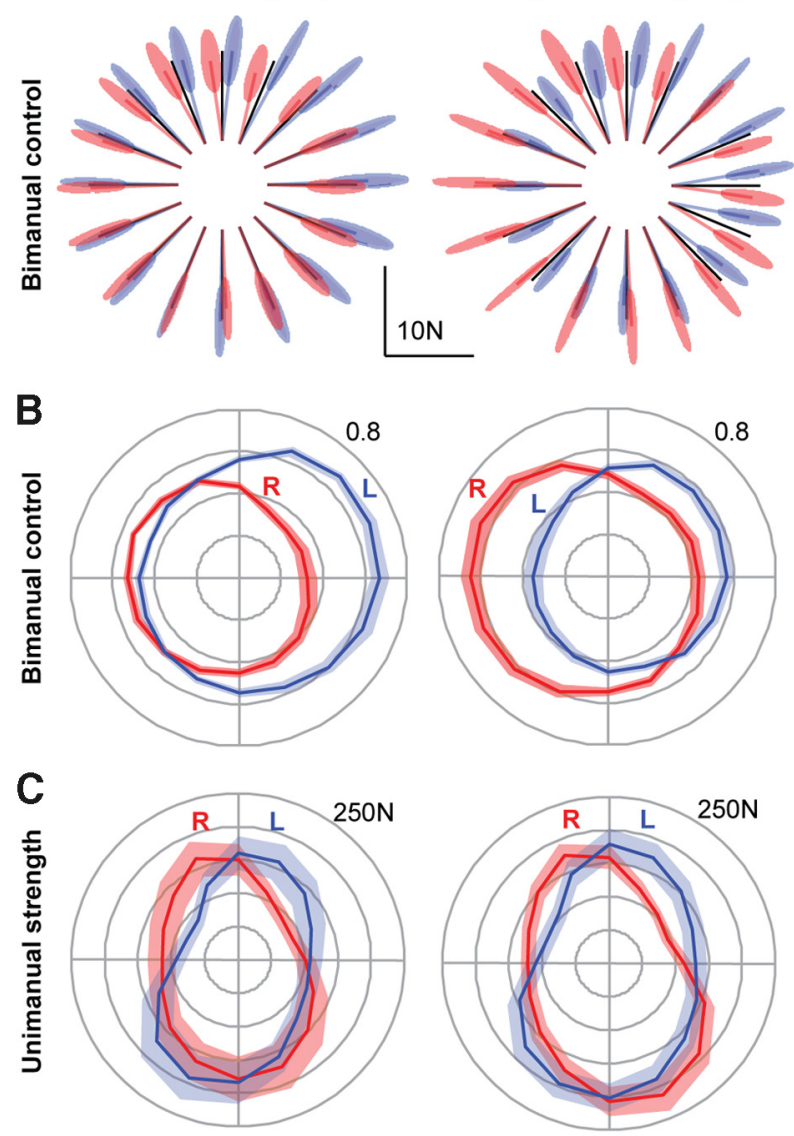

D
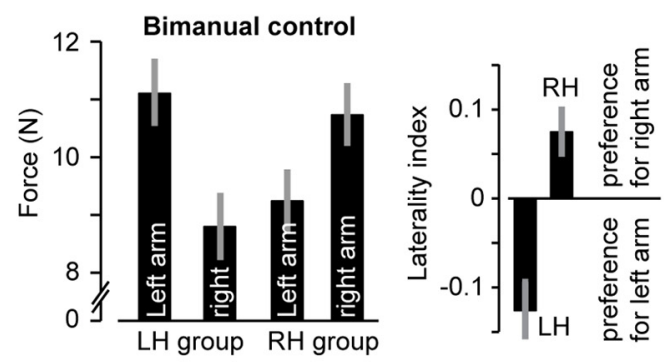

E

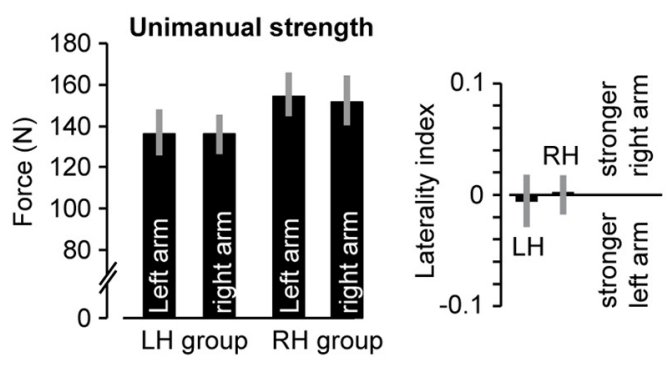

Figure 2. Bimanual control and unimanual strength in $\mathrm{LH}$ and $\mathrm{RH}$ young volunteers. $A$, The force vector produced by each arm, represented as a variance-covariance ellipse in which the center location corresponds to the mean of the force vector by each arm and the shading represents between-subject variance. $\boldsymbol{B}$, The force produced by each arm during bimanual control, represented as the fraction of total force produced by both arms, projected onto the target direction. Shading is between-subject SEM. C, Unimanual MVF as a function of direction. Shading is between-subject SEM. $\boldsymbol{D}$, Left plot, Force produced by each arm, averaged over all directions. Laterality index refers to the difference between the force produced by the right and left arms, divided by the sum of forces in the two arms (Eq. 9). Error bars indicate betweensubject SEM. E, Left plot, MVF produced by each arm, averaged over all directions. Laterality index refers to the difference between MVF produced by the right and left arms, divided by the sum of MVFs of the two arms. Error bars indicate between-subject SEM.
A Standard deviation of unimanual force

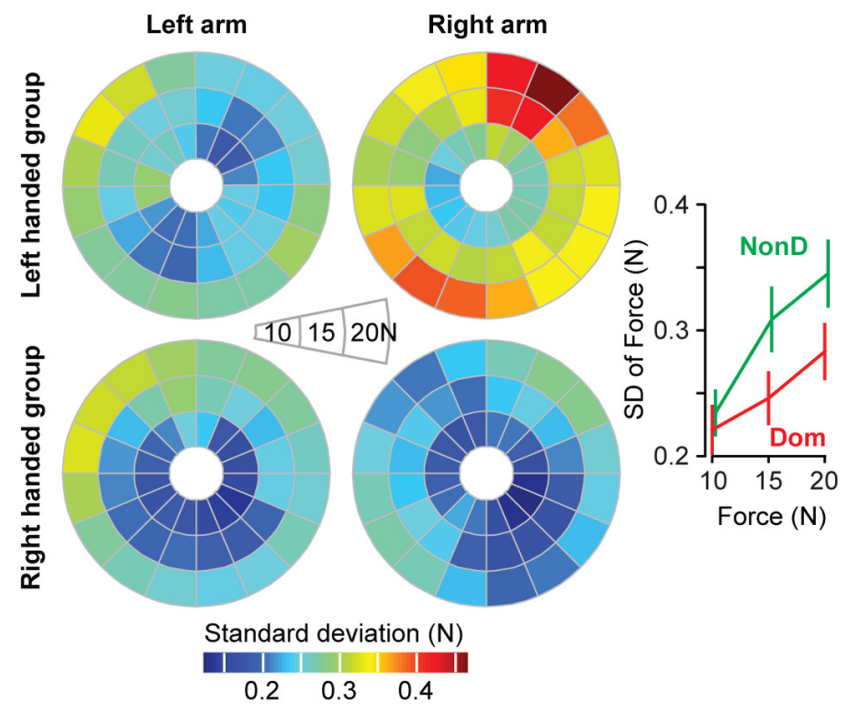

B
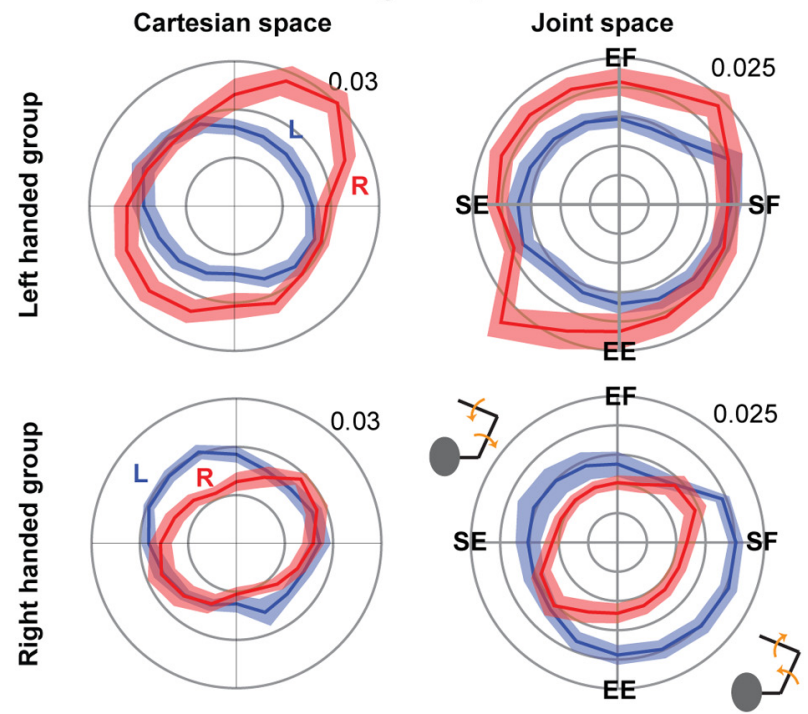

C
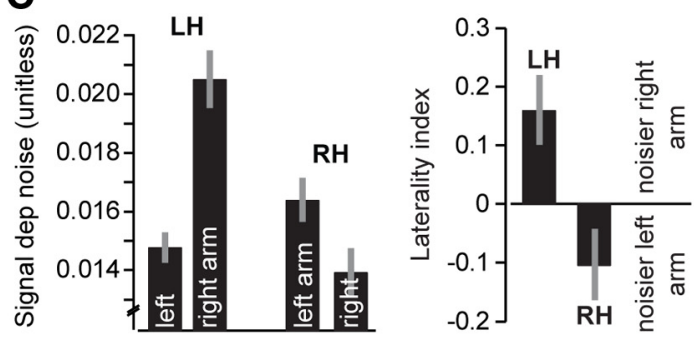

Figure 3. Unimanual noise patterns. $A, S D$ of force as a function of direction of force, measured at magnitudes of 10,15 , and 20N, for each arm and each group. Right subplot, SD of force as a function of force for the dominant (Dom) and the nondominant arm (NonD). This subplot was generated by collapsing the data for each subject across all directions and then combining the two groups. $\boldsymbol{B}$, Signal-dependent noise, measured as the slope of SD of force versus force magnitude, at each direction. Left column, This noise is plotted in Cartesian coordinates of force. Right column, The noise is plotted in joint coordinates of torque. SF, Shoulder flexion torque; EF, elbow flexion torque; SE, shoulder extension torque; EE, elbow extension torque. C, Left plot, Signal-dependent noise for each arm, averaged across all directions. Laterality index refers to the difference between signal-dependent noise produced by the right and left arms, divided by the sum of two arms. Data are mean \pm SEM. 
We further investigated the structure of signal-dependent noise in each arm by considering it in a more natural coordinate system, joint space (Fig. 3B, right column). For the LH group, noise in joint space appeared isotropic (ANOVA with direction as the repeated measure and arm as the group factor did not indicate a significant effect of direction, $\left.F_{(15,270)}=1.56, p>0.2\right)$. For the RH group, however, noise in joint space was direction-dependent (ANOVA with direction as the repeated measure and arm as the group factor, $\left.F_{(15,270)}=3.79, p<0.05\right)$. For the dominant arm of the $\mathrm{RH}$ group, the smallest noise was for torques produced in the shoulder extension/elbow flexion and shoulder flexion/elbow extension directions. Roughly speaking, for the right arm of the $\mathrm{RH}$ group, the noises were smallest when the hand was producing forces along an axis that ran from the hand to the shoulder.

\section{Unimanual noise and strength predict bimanual control}

If the two arms are equally strong, motor commands in a bimanual task should favor the arm that is less noisy. Can this idea account for behavior in the bimanual task? We considered a cost function (Eq. 4) in which, for each subject, motor commands were penalized based on the signal-dependent noise and MVF of each arm. Let us explain this cost briefly. If for a given direction the noise is large for a given arm, then the cost associated using that arm is high, discouraging its use. Similarly, if for a given direction, the arm can only produce a small MVF, then the cost associated using that arm is high, discouraging its use. The only free parameter in our model was the relative cost of noise for each arm, $a_{L}$ and $a_{R}$. This variable assigns a weight to the cost of noise, so that it could be compared with the weight associated with cost of strength $\left(1-a_{L}\right.$, etc.). Although the dominant arm was not generally stronger than the nondominant arm (Fig. 2E), the inclusion of MVF in the cost function is important because strength is direction-dependent (Fig. 2C).

We used each subject's noise and strength data to estimate the two parameters $a_{L}$ and $a_{R}$ for that subject. The forces produced by the model in the bimanual task are plotted in Figure 4A. In the LH group, the correlation between the direction-dependent modeled forces and measured values was $r=0.530, p=0.033$. In the $\mathrm{RH}$ group, the correlation between the modeled forces and measured values was $r=0.526, p=0.035$. The general correspondence between the model and data is summarized by the laterality index (Fig. 4B).

Next, we tested the hypothesis that regardless of handedness, a cost function that depended on unimanual measures of variance and effort could account for bimanual behavior. For this to be true, the cost function must not only have the same structure in the two groups, but also similar parameter values. That is, once we account for differences in unimanual variance and strength, there cannot be any other differences between the cost function in the LH and RH groups.

The cost of noise for each arm was estimated by $a_{L}$ and $a_{R}$. The values for these parameters are plotted in Figure $4 C$. We found that there was consistency in the way that the two groups penalized noise. The bimanual choices of the LH group were consistent with a large cost of noise in the right arm, whereas the bimanual choices of the RH group were consistent with a large cost of noise in the left arm. So if we now simply represent our cost function not in terms of left and right arms, but in terms of dominant and nondominant arms, there should be no difference between the parameter values of the two groups. To check for this, for each group we relabeled $a_{L}$ and $a_{R}$ as $a_{D}$ and $a_{N}$ (i.e., cost of noise in the dominant and nondominant arms). Indeed, ANOVA with a within-subject measure of $a_{D}$ and $a_{N}$, and between-subject factor
A Left handed group

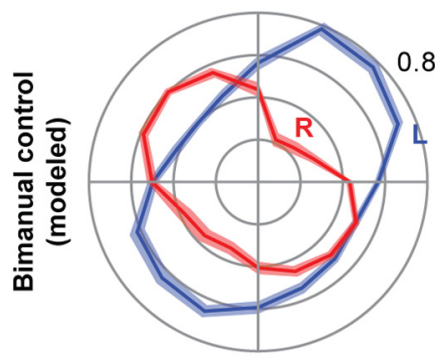
Right handed group

\section{B}

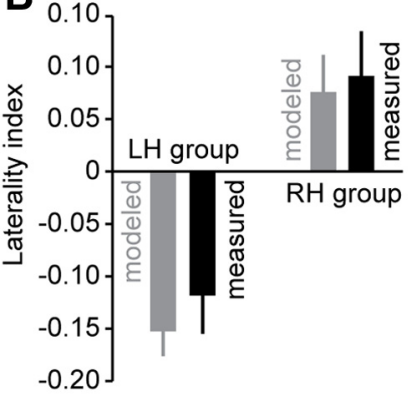

C Cost of variance

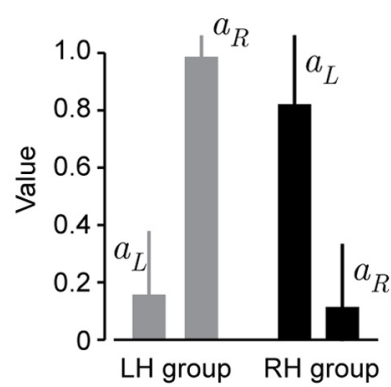

Figure 4. Model of bimanual control. $A$, For each subject, their unimanual MVF and signaldependent noise were used to model contribution of each arm during bimanual control (minimization of Eq. 4). Error bars indicate bootstrap estimated SD. B, Laterality index of the data produced by the model. Error bars for the model are SD; for the measured data, error bars indicate between-subject SEM. C, Distribution of model parameter values. In both groups, the coefficient that penalizes unimanual variance is larger for the nondominant arm. Error bars indicate bootstrap estimated SD of parameter values.

of handedness, produced no-significant effect of handedness $\left(F_{(1,18)}=1.57, p=0.23\right)$ and no interaction $\left(F_{(1,18)}=2.69, p=\right.$ $0.12)$, but of course a significant within-subject effect of dominant $\operatorname{arm}\left(F_{(1,18)}=17.1, p=0.0006\right)$. This demonstrates that the parameter values of the cost function did not differ between groups. Rather, in both groups, there was a high cost associated with noise in the nondominant arm (compared with its strength).

\section{Experiment 2: aging and bimanual control}

In young people, signal-dependent noise was direction-dependent and smaller in the dominant arm. We sought a new group of people in which this was not the case. In that group, we hoped to test a critical prediction of the hypothesis: if unimanual noise is comparable in the two arms, then in the bimanual task there should be no preference for the dominant arm.

We recruited a group of aged subjects. On the Edinburgh survey, they identified themselves as strongly RH (mean \pm SD score of $76 \pm 22$ for the aged RH group vs $88 \pm 13$ for the young RH group). Their unimanual strength and signal-dependent noise are plotted in Figure 5A. Strength of each arm in the aged group exhibited the same direction dependence that we had seen in the young RH group. However, ANOVA on the noise data with direction as the repeated measure, and left or right arm as the group factor revealed no significant effect of direction $\left(F_{(15,270)}=\right.$ 1.6, $p=0.21)$, no significant effect of arm $\left(F_{(1,18)}=0.005, p=\right.$ $0.94)$, and no interaction $\left(F_{(15,270)}=1.3, p=0.20\right)$. Therefore, in the aged group, unimanual noise was neither directiondependent nor arm-dependent. Both of these facts are in contrast to the young group (Fig. 5B).

The bimanual choices of the aged group are shown in Figure $5 C$. For ease of comparison, we also included in this figure the 
A Unimanual strength
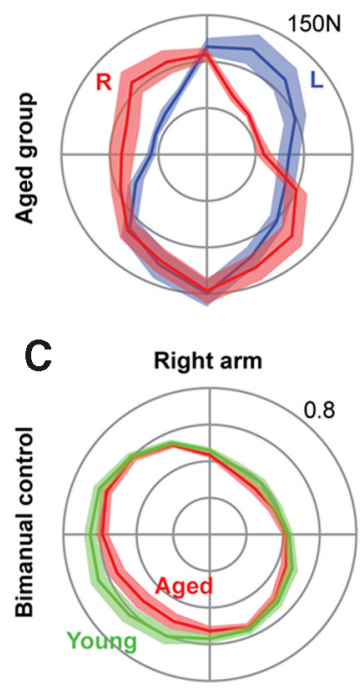

E

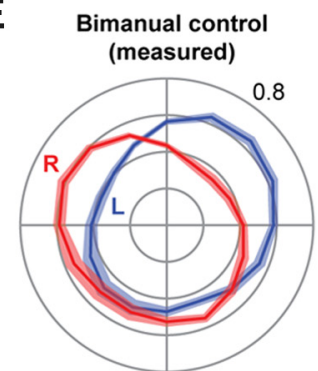

Unimanual signal dependent noise
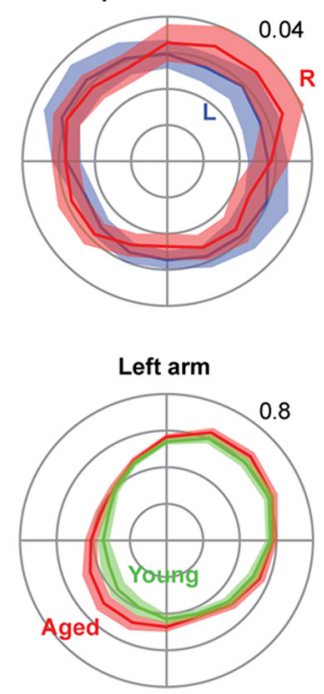

Bimanual control (modeled)

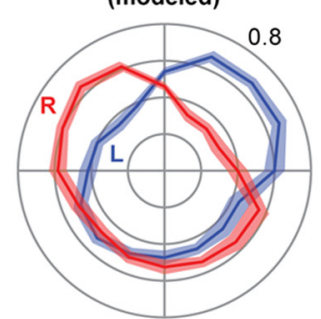

B

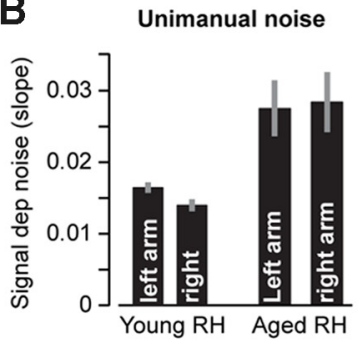

D
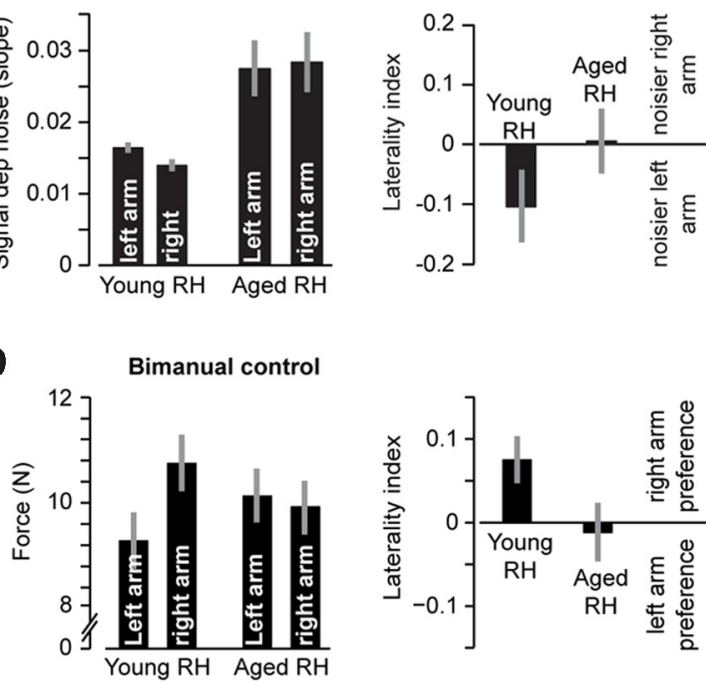

$\mathbf{F}$

Cost of variance

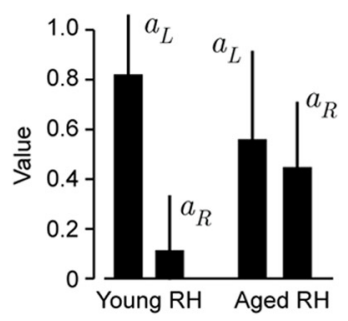

Figure 5. Performances of aged volunteers. $\boldsymbol{A}$, Unimanual MVF and signal-dependent noise. $\boldsymbol{B}$, Left plot, Signal-dependent noise for each arm, averaged across all directions. Laterality index refers to the difference between signal-dependent noise produced by the right and left arms, divided by the sum of two arms. C, Contributions of the left and right arms in bimanual control, plotted in the young RH volunteers as well as the aged. D, Left plot, Force produced by each arm, averaged over all directions. Laterality index refers to the difference between the force produced by the right and left arms, divided by the sum of forces in the two arms (Eq. 9). $\boldsymbol{E}$, Bimanual control in the aged group and the performance of the corresponding model. $\boldsymbol{F}$, Distribution of model parameter values. Data are mean \pm SEM.

choices made by the young RH group. In comparing the bimanual choices of the aged and young, it appeared that the aged group had reduced contributions of their right arm and increased contributions of their left arm. ANOVA on the difference between forces in the right and left arm with the within-subject measure of direction, and between-subject group of young and elderly, showed a significant effect of direction $\left(F_{(15,270)}=21.2, p<\right.$ $\left.10^{-5}\right)$, a trend toward significance for the effect of group $\left(F_{(1,18)}=\right.$ $3.75, p=0.067$ ), and no interaction. These data are summarized in Figure $5 D$. We saw a trend toward increased symmetry in the use of the two arms in the elderly subjects, although the difference between elderly and young was not statistically significant.

Figure $5 E$ plots the bimanual choices of the aged group. We used each subject's noise and strength data to estimate the cost of variance parameters $a_{L}$ and $a_{R}$. The model's choices in the bimanual task are also plotted in Figure 5E. The correlation between the direction-dependent modeled forces and measured values was $r=0.83, p<0.001$. The values of $a_{L}$ and $a_{R}$ for the aged and young $\mathrm{RH}$ groups are shown in Figure $5 F$. We found that the two groups differed in how they penalized variance. ANOVA with the within-subject repeated measure of $a_{L}$ and $a_{R}$, and the betweensubject factor of young or elderly, produced a significant effect of group $\left(F_{(1,18)}=5.7, p=0.029\right)$ and a significant interaction $\left(F_{(1,18)}=7.1, p=0.016\right)$. The interaction suggested that the younger group exhibited an asymmetric cost of noise for each arm, whereas the elderly group did not exhibit an asymmetry.

In summary, in the aged population, the noise in the two arms was comparable, and consistent with this, in the bimanual task they exhibited no preference for their dominant arm.

\section{Experiment 3: motor cortical stimulation and bimanual control}

We stimulated the motor cortex in the hope that this would alter bimanual control. Figure $6 A$ shows the effects of stimulation of the motor cortex in an $\mathrm{LH}$ subject and an $\mathrm{RH}$ young subject. In the sham condition, each subject had a preference for their dominant arm. When the anodal electrode was placed on the dominant M1 (and the cathode placed on the contralateral M1), both subjects increased their preference for their dominant arm. In contrast, when the cathodal electrode was placed on the dominant M1 (and the anode placed on the contralateral M1), we saw little or no change in the preference for the dominant arm. These data are summarized in Figure $6 B$. Based on these results, we chose to investigate the effects of anodal stimulation of the dominant M1 in a group of RH subjects.

We recruited a new group of young $\mathrm{RH}$ volunteers and applied the anodal electrode to the left motor cortex and the cathodal electrode to the right motor cortex. Bimanual behavior in the tDCS and sham conditions are shown in Figure 7A (top row). 
A
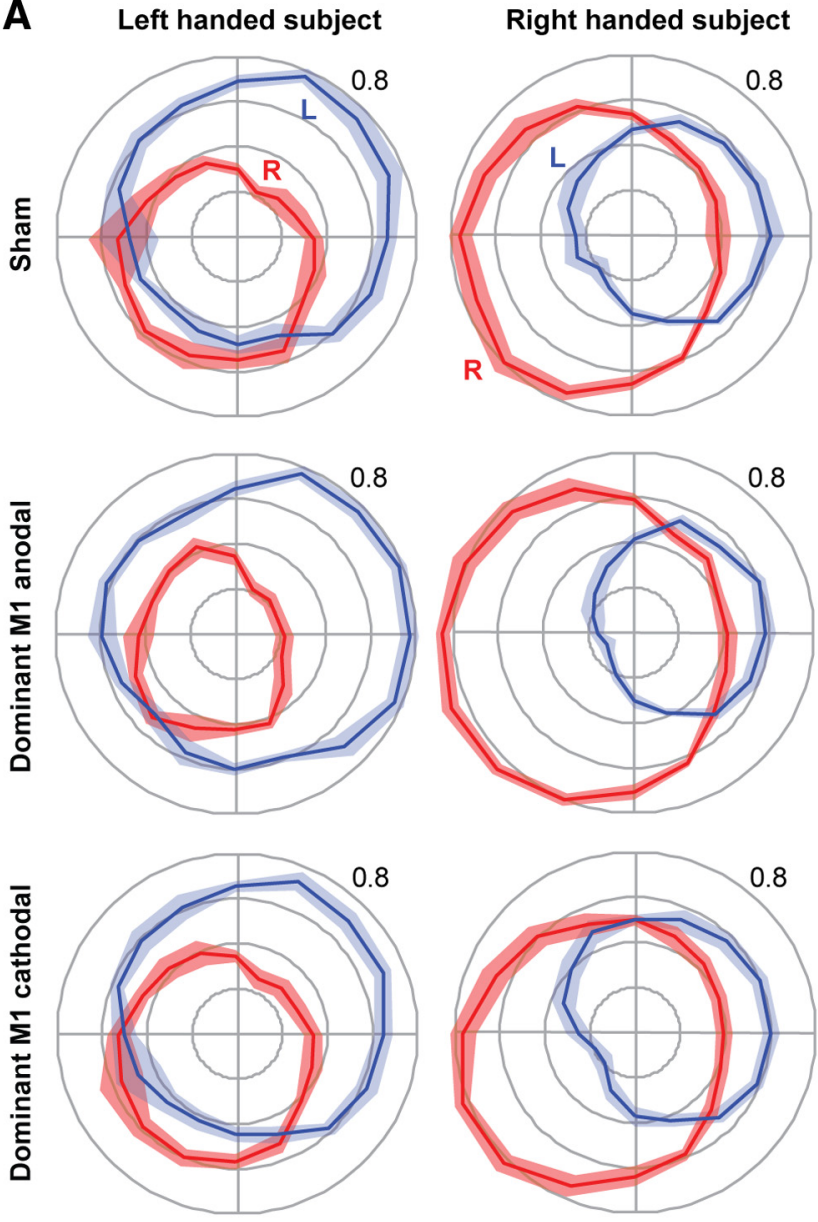

B

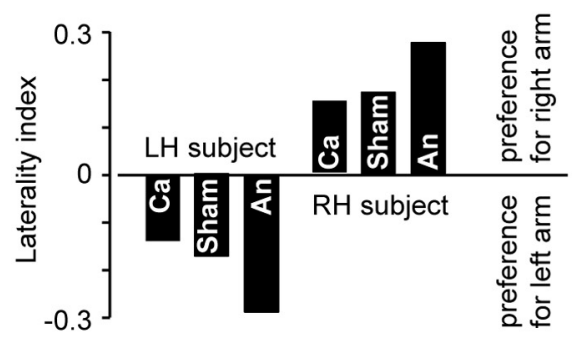

Figure 6. Anodal $\mathrm{tDCS}$ on the dominant hemisphere tends to increase the preference for the dominant arm in both $\mathrm{LH}$ and RH people. Data are from two subjects: one a RH individual and one a LH individual. Each subject participated in three experiments: sham, anodal tDCS of the dominant M1, and cathodal $\mathrm{DDCS}$ of the dominant M1. Stimulation is always bilateral, with one M1 serving as anode, whereas the other serves as cathode. $\boldsymbol{A}$, Behavior in the bimanual task. Each plot shows the fraction of force produced by each arm for each direction. $\boldsymbol{B}$, Laterality index for the three conditions in each subject. Ca, Cathodal stimulation of the dominant hemisphere; An, anodal stimulation of the dominant hemisphere.

tDCS produced an increase in the forces produced by the right arm, particularly for targets to the left. This resulted in a significant within-subject increase in the forces produced by the dominant arm with respect to the nondominant arm (Fig. $7 B$, top row, paired $t$ test, $\left.t_{(9)}=2.93, p=0.016\right)$. tDCS also produced an increase in the MVF of the dominant arm, particularly for targets to the top left and bottom right (Fig. $7 A$, middle row). We quantified this via a paired $t$ test of the average MVF of the right arm and found a significant increase in the tDCS condition $\left(t_{(9)}=\right.$ $3.62, p=0.006)$. There were no significant changes in the MVF of the left $\operatorname{arm}\left(t_{(9)}=1.39, p=0.20\right)$. Finally, tDCS did not produce a significant change in the unimanual noise patterns, although there was a trend toward reduction of noise in the dominant arm (Fig. 7B, bottom row). Therefore, anodal tDCS of the left motor cortex produced an increase in the strength of the right arm, as well as an increase in the use of the right arm during bimanual control.

\section{A model to relate unimanual noise with neural control}

Although the origin of signal-dependent noise in force production is poorly understood, the available evidence suggests that it is of neural origin (Jones et al., 2002), reflecting the number of neurons that are contributing to production of force (Galganski et al., 1993). Using a simple neuronal model (Eqs. 9-14), we used the signal-dependent noise measurements in each arm and each direction to estimate the distribution of neurons that may be contributing to production of force. In Figure $8 A$, we have plotted the model's predictions regarding the distribution of neurons for each arm (we have taken the liberty to label neurons to the hemisphere contralateral to the arm). To arrive at these results, we set $\mathrm{p}=1$ in Equation 14 and used the signal-dependent noise values for each group of subjects to estimate the number of neurons that contributed to each target force. For the RH young, the model predicted that a larger number of neurons were available in the left hemisphere to contribute to the task of force production with the contralateral arm, compared with the right hemisphere (Fig. $8 A$, top row). Furthermore, the distribution of these neurons was not isotropic, but greater for forces in the second and fourth quadrant (approximately aligned with the handshoulder axis). These patterns repeated in the LH young. However, in the aged group, the model suggested a large loss of neurons, particularly in the left hemisphere, compared with $\mathrm{RH}$ young.

An important question is whether the modeling and behavioral results have any correlates with neural control of force in the nervous system. During isometric force production, cells in the motor cortex are strongly modulated by the direction and magnitude of force (Sergio and Kalaska, 1998; Cabel et al., 2001), whereas this sensitivity is less in other frontal motor areas (Kakei et al., 2001) and much less in the posterior parietal cortex (Kalaska et al., 1990). Each cell has a preferred direction of reaching or force production for which it fires the most. Intriguingly, the distribution of preferred directions is anisotropic. For example, Scott et al. (2001) recorded from $214 \mathrm{M} 1$ neurons in 3 monkeys while maintaining the arm in the horizontal plane at a configuration close to that which we used (Fig. 1A). The animals made reaching movements to targets at various directions. The distribution of preferred direction of cells is reconstructed in Figure $8 B$ (left subplot). They found many more cells in the second and fourth quadrant than in the first and third quadrant. Our model's estimate of distribution of neurons is strongly correlated with these neurophysiological results: the correspondence between the distribution of modeled neurons in the left hemisphere of our young RH subjects and the left M1 of monkeys is $r=0.65$ $\left(F_{(1,14)}=10.46, p=0.006\right)$.

An important caveat is that the results of Scott et al. (2001) were from a reaching task and not an isometric force task. Sergio et al. (2005) compared activity of M1 cells in both tasks and found that, during the period near the start of the reach/force production, and during the period near reach completion and the subsequent hold interval, for a given cell the preferred directions in the two tasks are similar. Regardless, we considered another neurophysiological dataset. In an isometric task in which torques were applied to the shoulder and/or the elbow joints of two mon- 
A
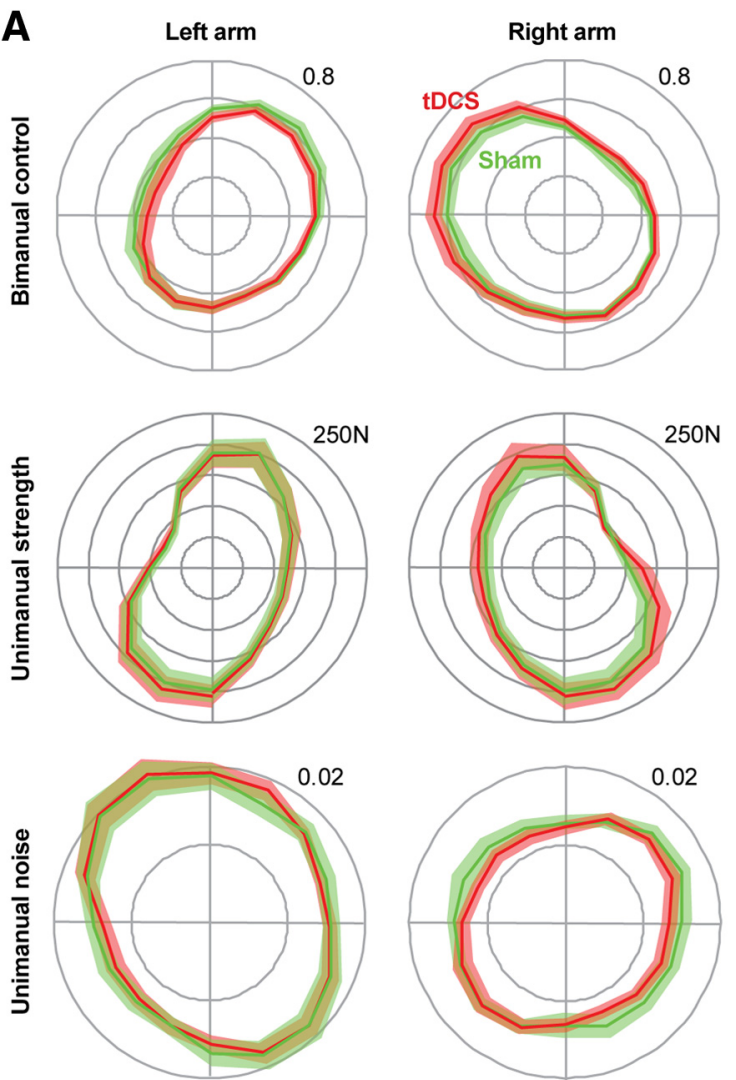

B
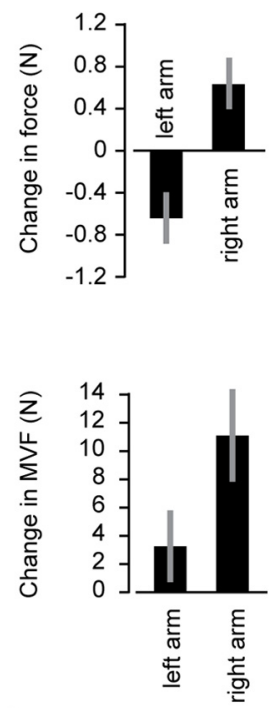

Figure 7. Effect of tDCS on bimanual control, unimanual strength, and noise of a group of RH young people. $A$, Top row, The force produced by each arm during bimanual control, represented as the fraction of total force produced by both arms. Middle row, MVF of each arm. Bottom row, Signal-dependent noise. $\boldsymbol{B}$, Top row, tDCS-induced change in force produced by each arm during bimanual control, averaged over all directions. Middle row, tDCS-induced change in MVF of each arm, averaged over all directions. Bottom row, tDCS-induced change in signal-dependent noise in each arm, averaged over all directions. Data are mean \pm SEM.

keys, Cabel et al. (2001) reported the preferred direction of torques that produced maximal activity in 88 cells in the arm area of the motor cortex. Their results are plotted in Figure $8 B$ (right subplot). There is an anisotropic distribution of preferred direction of cells, with most cells activated for torques that simultaneously engaged shoulder extension/elbow flexion or shoulder flexion/elbow extension. How does this correspond to our noise measurements? Using the joint space noise data in the right arm of our RH group (Fig. 3B), we estimated the number of neurons in each direction of torque. The model results are plotted in Figure $8 C$. We found that the predicted number of neurons was largest for shoulder extension/elbow flexion and shoulder flexion/ elbow extension, approximately consistent with the neurophysiological recordings.

Together, the results imply that, from the unimanual, directiondependent noise measurements from each arm, one may approximately estimate the size and distribution of preferred direction of neurons in the contralateral M1. Based on this assumption, our model predicts that handedness in young people is associated with a larger number of arm-related neurons in the dominant versus nondominant M1 (Fig. 8A). Compared with RH people, left handers may have fewer arm-related neurons in their dominant M1. With aging, there may be a reduction in the asymmetry of the number of arm-related neurons in the two motor cortices, possibly because of a strong reduction in the number of neurons in the dominant $\mathrm{M} 1$, but a weaker reduction in the nondominant $\mathrm{M} 1$.

\section{Discussion}

We considered a bimanual task in which people chose how much force to produce with each arm in order for the sum to equal a target amount. In Experiment 1, we found that the dominant arm of young people generally produced a greater force, but its contribution varied with direction of target. This was not because the dominant arm was stronger; rather, it was less noisy. Regardless of arm or handedness, the noise was smallest along an axis between the hand and the shoulder. Using an optimization framework, we found that a cost that included noise and strength of each arm accounted for both the direction- and handedness-dependent choices that people made. In this cost, noise in the nondominant arm was weighted more than in the dominant arm.

If unimanual noise is causally related to bimanual control, then people who have similar levels of unimanual noise in the two arms should show no preference for the dominant arm in bimanual control. In Experiment 2, we recruited a group of aged volunteers and found that unimanual noise was not different between the two arms, and bimanual control exhibited no preference for the dominant arm.

In Experiment 3, we applied anodal tDCS to the dominant motor cortex of $\mathrm{RH}$ young people. We found increased contributions of the dominant arm in bimanual control. Together, our results demonstrate that the motor commands that are sent by the brain to each arm during bimanual control are largely a reflection of the unimanual noise and strength of each arm.

Our study was inspired by work of O'Sullivan et al. (2009). In that work, predominantly $\mathrm{RH}$ young people produced isometric forces with fingers of their right and left hands to produce a target force. They found that noise was higher in the left hand of $\mathrm{RH}$ people and that a cost similar to the one we used here described bimanual control of hands. Together, the two reports demonstrate that unimanual noise and strength can account for bimanual control in both fingers and arms.

In our cost, forces were normalized with respect to strength and then penalized via a quadratic function. We chose the quadratic function because Fagg et al. (2002) had demonstrated its effectiveness in accounting for muscle activation patterns during isometric force production.

In our task, there was a contribution from each arm (i.e., burden sharing). However, the framework may be relevant to winner-take-all tasks. For example, $\mathrm{RH}$ people use their right arm to reach to stimuli on the right, left arm to reach to stimuli on the left, but prefer using their right arm to reach to stimuli that are straight ahead (Oliveira et al., 2010). The decision as to which act to perform is thought to be a race between two motor plans (Pastor-Bernier and Cisek, 2011). The plan that reaches threshold first is executed (Michelet et al., 2010). Coelho et al. (2013) demonstrated that the right arm displayed better performance 


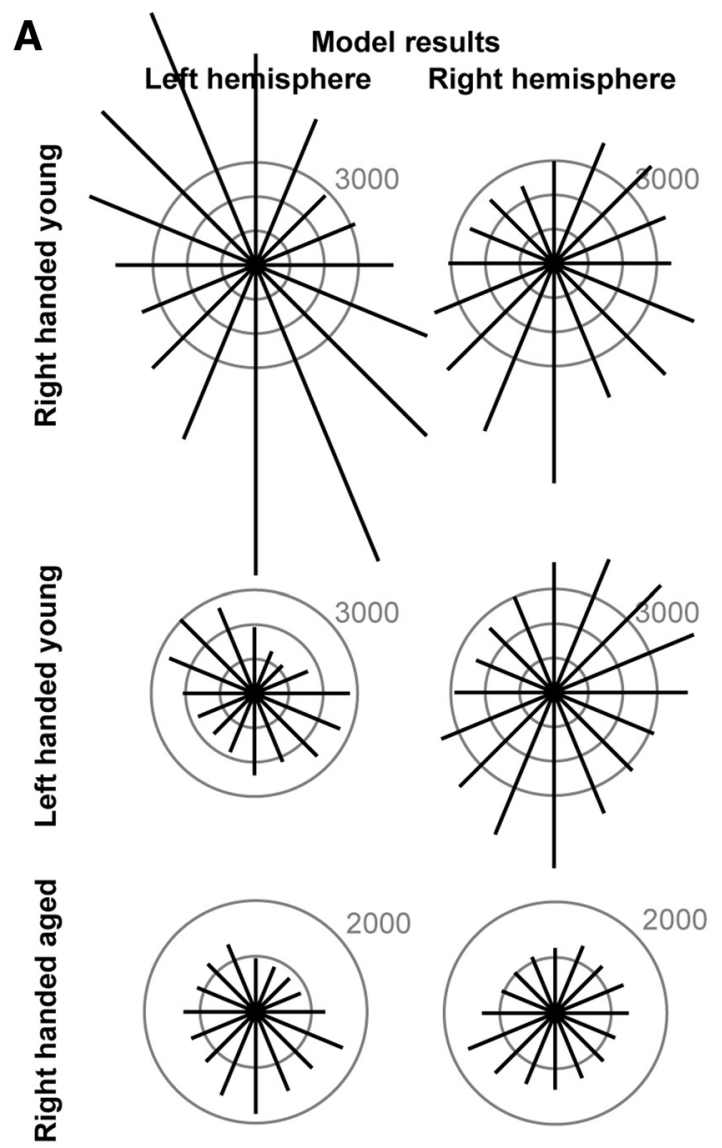

\section{B Left motor cortex of monkey \\ Isometric torque}
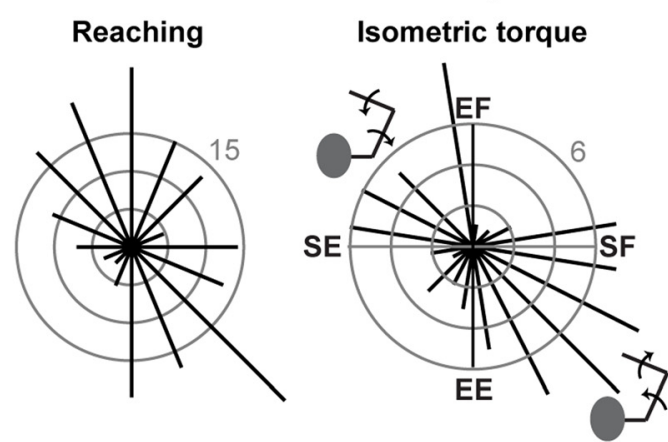

C

Model results

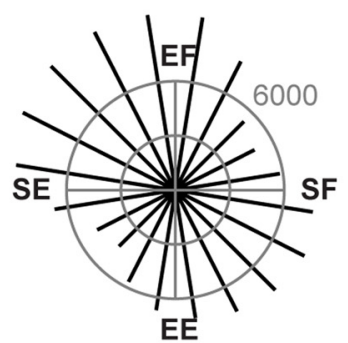

Figure 8. A model relating signal-dependent noise to distribution of neurons. $\boldsymbol{A}$, From the unimanual signal-dependent noise patterns in each arm (as represented in Cartesian space), we estimated the distribution of task-related neurons in the left and the right motor cortex for each force direction and each group. The number refers to number of cells. $\boldsymbol{B}$, Left, Distribution of preferred directions of neurons in the left M1 as recorded in 3 monkeys during reaching movements as reported previously (Scott et al., 2001). Right, Distribution of preferred direction of neurons in M1 as recorded in 2 monkeys during an isometric torque production task as reported previously (Cabel et al., 2001). C, From the unimanual signal-dependent noise patterns in the right arm of the RH group of subjects (as represented in joint space), we estimated the distribution of task-related neurons in the left M1 for each torque direction.

for the straight-ahead reaches than the left arm. This unimanual variance may affect the rate at which the plan for that arm proceeds to threshold. That is, bimanual preference in reaching, such as force production, may have its roots in a motor cost that depends on unimanual variance. Unimanual variance, in turn, may be a reflection of the number of task-relevant neurons in M1.

Control of the dominant arm is generally associated with less variability (Kalisch et al., 2006). Variance in production of force appears to be mainly the result of noise properties of neurons and not the muscles (Jones et al., 2002). In M1, a cortical region where cells are most sensitive to forces that are produced by the arm (Sergio and Kalaska, 1998; Cabel et al., 2001), SD of discharge grows linearly with the magnitude of discharge (Schwartz, 1994). A cost that penalizes variance favors a neural computation that spreads activations to as many cells as possible to keep their firing rates low. We observed that noise in the dominant arm was generally less than in the nondominant arm, which would imply that the number of neurons available to control the arm is larger in the dominant hemisphere. This conjecture is consistent with measures of M1 activation in RH and LH people (Volkmann et al., 1998; Bernard et al., 2011).

We found that unimanual noise was direction-dependent, and for some directions the dominant and nondominant arms were equally noisy (Fig. 3B). Using a model, we suggested that the shape of the noise function implied a specific anisotropy in the distribution of neurons that control force. We showed that the anisotropy in the distribution of modeled neurons corresponded to the distribution of preferred directions of cells in M1 (Scott et al., 2001): when the arm is in the horizontal plane, both the minimum of the noise function and the maximum of the density of preferred direction of cells in M1 lie on an axis between the hand and the shoulder. We found that the smallest variance in force was associated with elbow-flexion/shoulder-extension torques, and our model predicted that the largest number of neurons is associated with this direction of torque. Interestingly, when M1 is microstimulated, the stimulation is more likely to activate elbow flexors than extensors and more likely to activate shoulder extensors than flexors (Park et al., 2004). Together, it appears that unimanual noise in isometric force production may be a proxy for the density of preferred direction of cells in M1.

We observed that, during bimanual control, the cost of noise in the nondominant arm was more than in the dominant arm. Arm area of M1 has neurons that receive input from neurons in the contralateral M1 arm region (Muakkassa and Strick, 1979). The functional significance of this may be the observation that, during force production, one M1 inhibits the other (Morishita et al., 2012). Interestingly, this inhibition is greater from the dominant to the nondominant M1 than vice versa (Netz et al., 1995). If unimanual variance is associated with the number of neurons that are available to produce force in a given direction, then the greater cost of noise in the nondominant hemisphere during 
bimanual control implies that the dominant hemisphere recruits more neurons than would be predicted by the ratio of task-relevant neurons in the two hemispheres. Therefore, we predict that, during bimanual control, activation of taskrelevant neurons in the dominant M1 produces a strong inhibition of the nondominant M1, whereas activation of neurons in the nondominant M1 produces a weak inhibition of the dominant M1.

We found that aging altered motor control in distinct ways: first, aging produced a greater increase in the noise of the dominant arm than the nondominant arm. The increased noise resulting from aging has been previously reported (Galganski et al., 1993), but the asymmetric increase appears to be a novel finding. Second, aging removed the direction-dependent structure of noise and made it isotropic. Third, the cost of noise was asymmetric in youth between the arms but became symmetric with aging. The first observation suggests that with aging there is neuronal loss in $\mathrm{M} 1$, but this loss is greater for regions that control the dominant arm. The second observation suggests that, within a hemisphere, there is a greater loss of neurons among cells that have their preferred directions along the hand-shoulder axis than other directions. The third observation suggests that, whereas in young people intercortical inhibition is asymmetric, with aging it becomes symmetric. This last conjecture may be related to the fact that aging reduces the inhibition between the two M1s (Langan et al., 2010).

We stimulated the motor cortex because cells in M1 show strong sensitivity to the forces that are produced by the arm (Sergio and Kalaska, 1998; Cabel et al., 2001). Anodal tDCS increases the resting membrane potential of neurons, bringing them closer to a depolarized state, requiring less input to make the neuron fire (Purpura and McMurtry, 1965). This may explain our observation that, with anodal stimulation of the dominant hemisphere, the contribution of the dominant arm increased in bimanual control, along with its apparent strength. A limitation in our experiment is that we applied tDCS after sham stimulation, and therefore the order was not counterbalanced. This is a fundamental limitation of a single session experiment, as tDCS induces aftereffects (Nitsche and Paulus, 2000) that prevent a scenario in which sham is applied after termination of stimulation.

Whereas we observed that bimanual coordination across various people was associated with the noise of each effector, de Rugy et al. (2012) found that people could not learn new coordination when noise in a muscle was artificially changed. An interesting follow-up would be to use unimanual training to alter noise properties of an effector and then test whether this change alters voluntary behavior during bimanual control. Alternatively, it has been shown that short-term interventions, such as ischemic nerve block, can alter cortical representations associated with forearm flexors but not extensors (Vallence et al., 2012). These cortical changes should coincide with changes in voluntary behavior during bimanual control.

In conclusion, our study sheds light on how the nervous system solves redundancy in motor coordination, by identifying a central contribution of strength and variance of each effector. Variance in the ability to control an effector may be a reflection of the density of the neural representation of that effector in the motor cortex.

\section{References}

Bernard JA, Taylor SF, Seidler RD (2011) Handedness, dexterity, and motor cortical representations. J Neurophysiol 105:88-99. CrossRef Medline
Cabel DW, Cisek P, Scott SH (2001) Neural activity in primary motor cortex related to mechanical loads applied to the shoulder and elbow during a postural task. J Neurophysiol 86:2102-2108. Medline

Coelho CJ, Przybyla A, Yadav V, Sainburg RL (2013) Hemispheric differences in the control of limb dynamics: a link between arm performance asymmetries and arm selection patterns. J Neurophysiol 109:825-838. CrossRef Medline

Craigmile PF, Guttorp P, Percival DB (2005) Wavelet-based parameter estimation for polynomial contaminated fractionally differenced processes. IEEE Trans Sig Proc 53:3151-3161. CrossRef

de Rugy A, Loeb GE, Carroll TJ (2012) Muscle coordination is habitual rather than optimal. J Neurosci 32:7384-7391. CrossRef Medline

Fagg AH, Shah A, Barto AG (2002) A computational model of muscle recruitment for wrist movements. J Neurophysiol 88:3348-3358. CrossRef Medline

Galganski ME, Fuglevand AJ, Enoka RM (1993) Reduced control of motor output in a human hand muscle of elderly subjects during submaximal contractions. J Neurophysiol 69:2108-2115. Medline

Hoffman DS, Strick PL (1999) Step-tracking movements of the wrist: IV. Muscle activity associated with movements in different directions. J Neurophysiol 81:319-333. Medline

Jan Nijhof NE, Gabriel DA (2006) Maximum isometric arm forces in the horizontal plane. J Biomech 39:708-716. CrossRef Medline

Jones KE, Hamilton AF, Wolpert DM (2002) Sources of signal-dependent noise during isometric force production. J Neurophysiol 88:1533-1544. Medline

Kakei S, Hoffman DS, Strick PL (2001) Direction of action is represented in the ventral premotor cortex. Nat Neurosci 4:1020-1025. CrossRef Medline

Kalaska JF, Cohen DA, Prud'homme M, Hyde ML (1990) Parietal area 5 neuronal activity encodes movement kinematics, not movement dynamics. Exp Brain Res 80:351-364. Medline

Kalisch T, Wilimzig C, Kleibel N, Tegenthoff M, Dinse HR (2006) Agerelated attenuation of dominant hand superiority. PLoS One 1:e90. CrossRef Medline

Langan J, Peltier SJ, Bo J, Fling BW, Welsh RC, Seidler RD (2010) Functional implications of age differences in motor system connectivity. Front Syst Neurosci 4:17. CrossRef Medline

Michelet T, Duncan GH, Cisek P (2010) Response competition in the primary motor cortex: corticospinal excitability reflects response replacement during simple decisions. J Neurophysiol 104:119-127. CrossRef Medline

Mordillo-Mateos L, Turpin-Fenoll L, Millán-Pascual J, Núñez-Pérez N, Panyavin I, Gómez-Arguelles JM, Botia-Paniagua E, Foffani G, Lang N, Oliviero A (2012) Effects of simultaneous bilateral tDCS of the human motor cortex. Brain Stimul 5:214-222. CrossRef Medline

Morishita T, Uehara K, Funase K (2012) Changes in interhemispheric inhibition from active to resting primary motor cortex during a fine-motor manipulation task. J Neurophysiol 107:3086-3094. CrossRef Medline

Muakkassa KF, Strick PL (1979) Frontal lobe inputs to primate motor cortex: evidence for four somatotopically organized 'premotor' areas. Brain Res 177:176-182. CrossRef Medline

Netz J, Ziemann U, Hömberg V (1995) Hemispheric asymmetry of transcallosal inhibition in man. Exp Brain Res 104:527-533. Medline

Nitsche MA, Paulus W (2000) Excitability changes induced in the human motor cortex by weak transcranial direct current stimulation. J Physiol 527:633-639. CrossRef Medline

Oldfield RC (1971) The assessment and analysis of handedness: the Edinburgh inventory. Neuropsycholgia 9:97-113. CrossRef

Oliveira FT, Diedrichsen J, Verstynen T, Duque J, Ivry RB (2010) Transcranial magnetic stimulation of posterior parietal cortex affects decisions of hand choice. Proc Natl Acad Sci U S A 107:17751-17756. CrossRef Medline

O'Sullivan I, Burdet E, Diedrichsen J (2009) Dissociating variability and effort as determinants of coordination. PLoS Comput Biol 5:e1000345. CrossRef Medline

Park MC, Belhaj-Saif A, Cheney PD (2004) Properties of primary motor cortex output to forelimb muscles in rhesus macaques. J Neurophysiol 92:2968-2984. CrossRef Medline

Pastor-Bernier A, Cisek P (2011) Neural correlates of biased competition in premotor cortex. J Neurosci 31:7083-7088. CrossRef Medline

Purpura DP, McMurtry JG (1965) Intracellular activities and evoked poten- 
tial changes during polarization of motor cortex. J Neurophysiol 28:166185. Medline

Schwartz AB (1994) Direct cortical representation of drawing. Science 265: 540-542. CrossRef Medline

Scott SH, Gribble PL, Graham KM, Cabel DW (2001) Dissociation between hand motion and population vectors from neural activity in motor cortex. Nature 413:161-165. CrossRef Medline

Sergio LE, Kalaska JF (1998) Changes in the temporal pattern of primary motor cortex activity in a directional isometric force versus limb movement task. J Neurophysiol 80:1577-1583. Medline

Sergio LE, Hamel-Pâquet C, Kalaska JF (2005) Motor cortex neural correlates of output kinematics and kinetics during isometric-force and armreaching tasks. J Neurophysiol 94:2353-2378. CrossRef Medline

Shadmehr R, Mussa-Ivaldi S (2012) Biological learning and control: how the brain builds representations, predicts events, and makes decisions. Cambridge, MA: MIT.

Shim JK, Olafsdottir H, Zatsiorsky VM, Latash ML (2005) The emergence and disappearance of multi-digit synergies during force-production tasks. Exp Brain Res 164:260-270. CrossRef Medline

Vaillancourt DE, Newell KM (2003) Aging and the time and frequency structure of force output variability. J Appl Physiol 94:903-912. CrossRef Medline

Vallence AM, Hammond GR, Reilly KT (2012) Increase in flexor but not extensor corticospinal motor outputs following ischemic nerve block. J Neurophysiol 107:3417-3427. CrossRef Medline

Volkmann J, Schnitzler A, Witte OW, Freund H (1998) Handedness and asymmetry of hand representation in human motor cortex. J Neurophysiol 79:2149-2154. Medline 